\title{
E-Learning: Content allein reicht nicht
}

\section{E-Learning nimmt viele Formen an: im Trend liegen Podcasts und Kurzvideos für Microlear- ning. Sie werden sowohl von Unternehmen als auch von Schulen und Hochschulen zuneh- mend verwendet. Wo kommt der Content her?}

Wie verschaffen Sie sich am liebsten einen Überblick über ein Thema: durch Anschauen eines Videos, Hören eines Podcasts oder Lesen eines mehrseitigen Dokuments? Wenn es um Wissensvermittlung und Bildung geht, bevorzugen viele Menschen Online-Angebote. Und da heute jeder mit einem Smartphone ausgestattet ist, finden Lernprozesse an jedem Ort, zu jeder Zeit statt. Wissen wird überall konsumiert, auch unterwegs.

Erklärfilme und Kurzvorträge à la TEDx, Audio-Podcasts und auch AR („augmented reality“) haben sich als sinnvolle Ergänzung für das E-Learning etabliert und werden sowohl in Unternehmen als auch in Schulen und Hochschulen verwendet. Sie eignen sich bestens für den Wissenstransfer in kleinen Lerneinheiten. Aber auch komplexe Inhalte lassen sich schneller, anschaulicher und damit effizienter vermitteln als etwa mit Präsentationsfolien und langen Texten im Wiki.

Dieser Content will verwaltet, bearbeitet und zur Verfügung gestellt werden. Gefragt sind Technologien, welche die Handhabung vereinfachen und effizienter gestalten: wie Contentmanagement- und Media-Asset-Managementsysteme. Sie erleichtern die Organisation der (Lern-)Medien und vereinfachen die Recherche und das Auffinden von Inhalten. Nicht umsonst zählen viele Schulbuch- und Fachbuchverlage bzw. Bildungsmedienanbieter wie z. B. Cornelsen, Klett oder Hueber zu den langjährigen Kunden von SIX. Sie benötigen flexible und leistungsfähige Technologien, die vielfältige und heterogene Szenarien unterstützen - von Lernkatalogen über Online-Tests bis hin zu „packaged content“. Mithilfe der Content-Management-Technologie werden zum Beispiel Kommunikations- und Informationsplattformen geschaffen, die den standortunabhängigen Zugriff auf zentrale Daten und Informationsdienste gewährleisten. Das Medien-Asset-Managementsystem sorgt seinerseits für die effiziente Steuerung der Prozesse rund um Bildbeschaffung und Bildauswahl.

Experten aus der Bildungsbranche sind der Meinung, dass in den nächsten Jahren Erklärfilme sowie Blended-Learning-

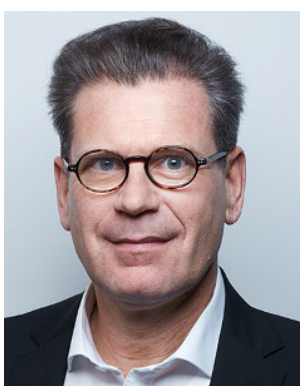

Martin Hippe ( $\triangle)$

ist seit Anfang Oktober 2019 Geschäftsführer der Six Offene Systeme GmbH. Hüppe blickt auf eine lange Erfahrung in der IT-Branche und im Verlagsumfeld zurück. Er war Geschäftsführer beim Bildungsmedienanbieter Cornelsen und dem Ernst Klett Verlag, beim Bündnis für Bildung e.V. sowie dem Didacta Verband e.V. Zuletzt leitete er die Firma IServ, dem Anbieter von Schulservern. Zur Person: https://www.six.de/unternehmen/ news-und-presse/erweiterung-dergeschaeftsleitung-bei-six presse@six.de

Six Offene Systeme GmbH Am Wallgraben 99, 70565 Stuttgart, Deutschland

Angebote im Bereich E-Learning kommerziell besonders erfolgreich sein werden. Für den Erfolg der neuen Lernszenarien und Methoden - online oder hybrid (Blended Learning), synchron oder asynchron - ist die schnelle und zuverlässige Verfügbarkeit des Contents entscheidend. 